\title{
Surface Study of ZnO Thin Film using Image Processing Techniques
}

\author{
G.R.Kandhasamy \\ Research Scholar \\ Department of Electronics and \\ Instrumentation \\ Bharathiar University, \\ Coimbatore-46
}

\author{
S.Muruganand, $\mathrm{PhD}$ \\ Assistant Professor \\ Department of Electronics and \\ Instrumentation \\ Bharathiar University, \\ Coimbatore-46
}

\author{
M.Pavithra \\ Guest Lecturer \\ Department of Electronics and \\ Instrumentation \\ Bharathiar University, \\ Coimbatore-46
}

\begin{abstract}
Surface study of thin film is characterized by employing scanning Electron microscopy (SEM). The implementation of image processing algorithm is to determine the pores in the SEM images of thin film. Image segmentation is applied to detect the pores in the thin film. In this paper, $\mathrm{ZnO}$ thin film is used for the surface study by various edge detection techniques. As the result canny edge detection gives the best porosity information of $\mathrm{ZnO}$ thin film SEM images. This result is taken by performing the statistical analysis (ie) finding the PSNR (Peak Signal to Noise Ratio) values .This may help to identify the particular place of pores and pits in thin film SEM images.
\end{abstract}

\section{Keywords}

Thin films, SEM images, Image processing, Segmentation, PSNR values, canny edge detection

\section{INTRODUCTION}

Scanning Electron Microscope (SEM) is very useful technique to acquire the high magnification images of the specimens. Secondary electron is predominately used for surface property studies of the material under SEM technology. Thin film technology is availed by many of the modern fields like MEMS, Nano technology etc [11]. Nowadays thin films are used to develop the solar cells. The quality of light reflection depends on the surface of the thin films [1]. Optical thin films are mostly used in automotive industry for non-contact measurement of surfaces. This can be achieved by observing the SEM images [2]. SEM images are used to study the strain measurement of the thin films with the help of image processing techniques [3]. PSNR is applied to develop the efficiency of the image segmentation results. In this paper, we discuss the surface property of the $\mathrm{ZnO}$ thin film by image segmentation (i.e. one of the image processing techniques). The objective result of the various edge detection images are displayed and discussed in this paper.

\section{MATERIALS AND METHOD}

Image processing techniques for finding the porosity consist of Image Acquisition, Image Enhancement, Image Segmentation and Feature extraction. The below block diagram shows the proposed approach. The thin films were analyzed and examined using Scanning Electron microscopy. An SEM generates high energy electrons and focuses them on a specimen. The thin films were subjected to secondary electron imaging in SEM produces vivid images with good resolution $1-5 \mu \mathrm{m}[4,5]$. The images from the SEM are in indexed mode [6] so that these images should be converted to gray scale mode. The process of conversion of a gray scale

image into a binary is called thresholding. In a binary image, object pixels are represented by $1 \mathrm{~s}$ or the highest intensity possible in the image.

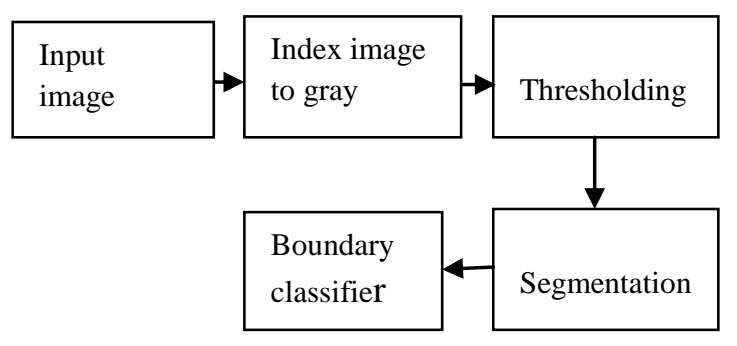

Figure 1: Block diagram of proposed method

\subsection{EDGE DETECTION}

\subsubsection{Sobel Operator}

The operator consists of a pair of $3 \times 3$ convolution kernels as shown in Figure 1. One kernel is simply the other rotated by $90^{\circ}$.

$$
\begin{array}{lll}
-1 & 0 & +1 \\
-2 & 0 & +2 \\
-1 & 0 & +1
\end{array}
$$

$\mathrm{G}_{\mathrm{x}}$

Figure 1: Masks used by Sobel Operator

These kernels are designed to respond maximally to edges running vertically and horizontally relative to the pixel grid, one kernel for each of the two perpendicular orientations. The kernels can be applied separately to the input image, to produce separate measurements of the gradient component in each orientation (call these $G x$ and $G y$ ). These can then be combined together to find the absolute magnitude of the gradient at each point and the orientation of that gradient [8]. The gradient magnitude is given by:

$$
|G|=\sqrt{G x^{2}+G y^{2}}
$$

Typically, an approximate magnitude is computed using:

$$
|G|=|G x|+|G y|
$$

which is much faster to compute. The angle of orientation of the edge (relative to the pixel grid) giving rise to the spatial gradient is given by:

$$
\theta=\arctan \left(\frac{G y}{G x}\right)-3 \pi / 4
$$




\subsubsection{Roberts edge detection}

The Roberts Cross operator performs a simple, quick to compute, 2-D spatial gradient measurement on an image. Pixel values at each point in the output represent the estimated absolute magnitude of the spatial gradient of the input image at that point. The operator consists of a pair of $2 \times 2$ convolution kernels as shown in Figure 2. One kernel is simply the other rotated by $90^{\circ}$. This is very similar to the Sobel operator $[7,8]$.

$\begin{array}{llrl}1 & 0 & 0 & 1 \\ 0 & -1 & -1 & 0 \\ \text { Gx } & \text { Gy }\end{array}$

Figure 2: Mask used by Roberts operator

\subsubsection{Prewitt edge detection}

Prewitt operator edge detection masks are the one of the oldest and best understood methods of detecting edges in images. The strength of the edge at given location is then the square rote of the sum of the squares of two derivatives [8].

\subsubsection{Canny edge detection}

The Canny edge operator takes the grayscale image as input and calculates the gradient of intensities, a vector with both magnitude and direction. It is applied to find both the strong and weak edges of each particle. The normal of the edge aligns with the direction of the gradient vector, and the magnitude gives the strength of the edge. Canny edge detection uses linear filtering with a Gaussian kernel to smooth noise and then computes the edge strength and direction for each pixel in the smoothed image. This is done by differentiating the image in two orthogonal directions and computing the gradient magnitude as the root sum of squares of the derivatives. The gradient direction is computed using the arctangent of the ratio of the derivatives. Edge pixels are identified as the pixels that survive a thinning process called non-maximal suppression. In this process, the edge strength of each edge pixel is set to zero if its edge strength is not larger than the edge strength of the two adjacent pixels in the gradient direction [7].

The following algorithms are used in canny edge detection

1. Smooth the image by convolving with a Gaussian of variance $\sigma^{2}$.

2. Compute the gradient of the smoothed image, and compute its magnitude and direction.

3. Non-maximal suppression: Select the pixels where the gradient magnitude has a local maximum in the direction of the gradient.

4. Using two specified thresholds, $\mathrm{T} 1$ and $\mathrm{T} 2$, with $\mathrm{T} 1<\mathrm{T} 2$, mark selected pixels with gradient magnitude larger than $\mathrm{T} 2$ as 'strong', and pixels with magnitude between $\mathrm{T} 1$ and $\mathrm{T} 2$ as 'weak'.

5. Select all strong pixels, and all weak pixels that are connected to strong pixels horizontally, vertically or diagonally.

\subsubsection{Statistical measures}

The first measurement used in image processing is the mean square error (MSE) $[9,10]$.

$$
M S E=\frac{1}{M N} \sum_{(i, j \in X)}|A(i, j)-B(i, j)|^{2}
$$

$\mathrm{M}, \mathrm{N}$ is the dimensions of the image. Lower value of the MSE denotes the less error and PSNR becomes high because the MSE has the inverse relation with the PSNR.

$$
\operatorname{PSNR}(A, B)=10 \frac{\log _{10} R^{2}}{\operatorname{MSE}(A, B)}
$$

PSNR value is high for the good result images.

\section{EXPERIMENTAL WORK}

The thin film has been grown from solution by dip coating method at $400^{\circ} \mathrm{C}$ temperature. The pores of the $\mathrm{ZnO}$ thin films are studied using image processing.

\subsection{SETTINGS}

Those thin films are subjected to Secondary electron imaging in scanning electron microscopy under $5 \mathrm{keV}$ beam energy at a working distance of $500 \mu \mathrm{m}$. The SEM is saved as graphic file format for further process in MATLAB environment. The image acquisition is done by using 'imread' MATLAB command and it is converted into intensity to gray image. Thesholding is taken by converting the gray image into binary image. 2D adaptive filter is introduced to reduce the noise in the SEM images [4]. And this filtered image is further processed by introducing various edge detection techniques. After applying edge detection to the SEM image, quantitative measurement is taken using PSNR measures.

\section{RESULTS}

We apply our method to SEM images. Our intention is to find the pores of the thin films. Figure (1a) shows the graphics format of the thin film SEM image and figure (1b) shows the filtered image of the $\mathrm{ZnO}$ thin films.

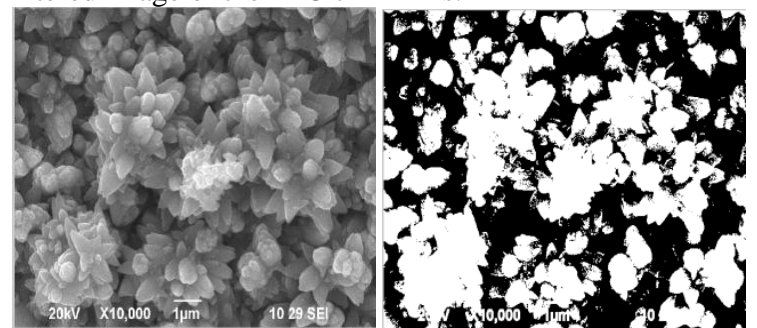

1(a) Original image

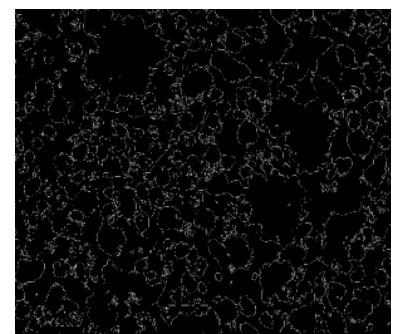

2(a) 'canny' edge detection 1(b) Filtered image

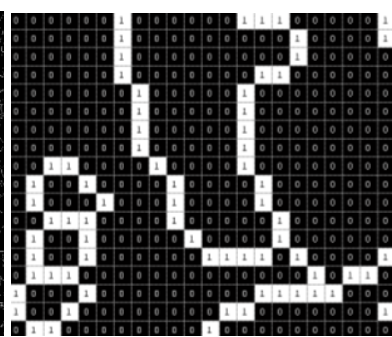

2(b) Pixel region
Figure (2a) and (2b) shows the edge detected image and corresponding pixel region of the thin film. After filtering the image those images are subjected to edge detected image shows the pixel regions of the pores as ones. From this result 
the pores of the $\mathrm{ZnO}$ thin films are found using canny edge detection.

Table 1: Statistical Measures

\begin{tabular}{|r|l|l|}
\hline S.No. & Edge Detectors & PSNR values \\
\hline 1. & Sobel & $+2.38 \mathrm{~dB}$ \\
\hline 2. & Roberts & $+2.27 \mathrm{~dB}$ \\
\hline 3. & Prewitt & $+2.37 \mathrm{~dB}$ \\
\hline 4. & Canny & $+2.40 \mathrm{~dB}$ \\
\hline
\end{tabular}

From the above table canny edge detectors gives the better results.

\section{CONCLUSION}

The microstructure plays vital role in thin films. The pores of the $\mathrm{ZnO}$ thin films determine the formation and growth of the thin films. In that case detecting the pores is very important in thin films. Canny detection gives the best result through statiscal measures to find the regions of the pores in the $\mathrm{ZnO}$ thin films.

\section{ACKNOWLEDGMENT}

We thank our Department Staff members for providing necessary facilities for carrying out this work.

\section{REFERENCES}

[1] A . Lin, J. Phillips, "Optimization of random diffraction gratings in thin-film solar cells using genetic algorithms" Solar Energy Materials \& Solar Cells 92 (2008) 1689 1696.

[2] C.-L. Tien et al. "Surface flatness of optical thin films evaluated by gray level co-occurrence matrix and entropy"Applied Surface Science 254 (2008) 4762-4767.

[3] W.N. Sharpe et.al. "Strain Measurements of Silicon Dioxide Micro specimens by Digital Imaging
Processing" Experimental Mechanics (2007) 47:649658.

[4] M.Varadhalakshmi et.al.," Grain boundary detection of the crystals using canny edge detection" I.J.E.M.S., Vol.3(2) 2012: 105 - 108.

[5] Fei Liu, Junshu Wu, Kunfeng Chen and Dongfeng Xue "Morphology study by using scanning electron microscopy", Microscopy: Science, Technology, Applications and Education, pp. 1781-1792, 2010.

[6] Thomas Shea et.al., " SEM image processing with Photoshop", Dept. of Geological Sciences, University of Hawaii and Oregon, USA.

[7] Michael D. Health et.al., "A Robust Visual Method for Assessing the Relative Performance of EdgeDetection Algorithms", IEEE transactions on Pattern analysis and Machine Intelligence, Vol.19,No.12, 1997.

[8] J. Matthews. "An introduction to edge detection: The sobel edge detector," Available at http://www.generation5.org/content/2002/im01.asp, 2002.

[9] Cao Yun-Fei et.al., "Multi-Level threshold image segmentation based on PSNR using artificial bee colony algorithm", Research Journal of Applied Sciences, Engineering and Technology 4(2): 104-107, 2012

[10] Zimin et al."Investigations of the pore formation in the leadselenide films using glacial acetic acid- and nitric acid-based electrolyte" Nanoscale Research Letters 2012, $7: 338$

[11] D. Van der Weken et al. "Combining neighbourhoodbased and histogram similarity measures for the design of image quality measures" Image and Vision Computing 25 (2007) 184-195 